\title{
Impairment of autophagy in scrapie-infected transgenic mice at the clinical stage
}

\author{
Óscar López-Pérez ${ }^{1,2}$ Janne Markus Toivonen ${ }^{1,3} \cdot$ Alicia Otero $^{2} \cdot$ Laura Solanas $^{1} \cdot$ Pilar Zaragoza $^{1,3}$. \\ Juan José Badiola ${ }^{2} \cdot$ Rosario Osta ${ }^{1,3} \cdot$ Rosa Bolea ${ }^{2} \cdot$ Inmaculada Martín-Burriel $\mathbb{D}^{1,2}$
}

Received: 9 May 2019 / Revised: 23 July 2019 / Accepted: 25 July 2019 / Published online: 2 September 2019

(c) United States \& Canadian Academy of Pathology 2019

\begin{abstract}
Autophagy appears to play a role in the etiology and progress of misfolded protein disorders. Although this process is dysregulated in prion diseases, it is unknown whether this impairment is a cause or a consequence of prion neuropathology. The study of autophagy during the progress of the disease could elucidate its role. For this purpose, we have investigated its regulation at different stages of the disease in $\mathrm{Tg} 338$ mice, a transgenic murine model that overexpresses the highly susceptible ovine VRQ prion protein allele. Mice were intracerebrally inoculated with mouse-adapted classical scrapie and euthanized at the preclinical and clinical stages of the disease. Regulation of autophagy was investigated analyzing the distribution of LC3-B and p62 proteins by immunohistochemistry. Moreover, the expression of genes involved in autophagy regulation was quantified by real-time PCR. LC3-B and p62 proteins were downregulated and upregulated, respectively, in the central nervous system of infected mice with clinical signs of scrapie. Accumulation of p62 correlated with scrapierelated lesions, suggesting an impairment of autophagy in highly prion-affected areas. In addition, Gas5 (growth arrestspecific 5), Atg5 (autophagy-related 5), and Fbxw7 (F-box and WD repeat domain containing 7) transcripts were downregulated in mesencephalon and cervical spinal cord of the same group of animals. The impairment of autophagic machinery seems to be part of the pathological process of scrapie, but only during the late stage of prion infection. Similarities between Tg338 mice and the natural ovine disease make them a reliable in vivo model to study prion infection and autophagy side by side.
\end{abstract}

\section{Introduction}

Prion diseases, also known as transmissible spongiform encephalopathies (TSEs), are fatal infectious neurodegenerative disorders that affect both humans and animals [1].

Supplementary information The online version of this article (https:// doi.org/10.1038/s41374-019-0312-z) contains supplementary material, which is available to authorized users.

$\square$ Inmaculada Martín-Burriel

minma@unizar.es

1 Laboratorio de Genética Bioquímica (LAGENBIO), Universidad de Zaragoza, IA2, IIS Aragón, 50013 Zaragoza, Spain

2 Centro de Encefalopatías y Enfermedades Transmisibles Emergentes, Universidad de Zaragoza, IA2, IIS Aragón, 50013 Zaragoza, Spain

3 Centro de Investigación Biomédica en Red sobre Enfermedades Neurodegenerativas (CIBERNED), Madrid, Spain
They include the various forms of Creutzfeldt-Jakob disease in humans, bovine spongiform encephalopathy in cattle, and scrapie in sheep and goats, which is one of the first identified TSEs [2]. The causative agents of TSEs are prions, misfolded isoforms of the physiological cellular prion protein $\left(\mathrm{PrP}^{\mathrm{c}}\right)$, named $\operatorname{PrP}$ "scrapie" $\left(\mathrm{PrP}^{\mathrm{Sc}}\right)$ [1]. Prions accumulate into the central nervous system (CNS), which is believed to be the main pathogenic event responsible for the pathological changes produced in TSE patients, including spongiform degeneration, vacuolization, glial activation, and neuronal loss [3, 4]. Prion diseases share common neurodegenerative features with other protein-misfolding diseases like Alzheimer's (AD), Huntington's (HD), and Parkinson's (PD) diseases [5]. However, the underlying pathomolecular mechanisms of this heterogeneous group of diseases are incompletely understood.

Autophagy is one of the most relevant protein homeostasis systems, and is involved in the degradation of damaged organelles and altered proteins from the cell via the lysosomal pathway [6]. In autophagic degradation, a 
double-membraned vacuole called autophagosome engulfs the cytoplasmic cargo and eventually fuses with lysosomes to form autophagolysosomes, where hydrolytic enzymes digest and recycle the luminal content [7]. Autophagic activity requires multiple proteins, such as MAP1LC3 (microtubule-associated protein 1 light chain 3), or LC3, and SQSTM1 (sequestosome 1), also known as p62. Whereas LC3 is tightly associated to the autophagosomal membrane and is therefore considered a reliable marker of autophagy [8], p62 is an important cargo receptor located throughout the cell and specifically degraded by autophagy [9]. Consequently, the evaluation of p62 accumulation has become a useful assay to estimate defects in autophagic degradation [8-11].

Dysfunction of the autophagic-lysosomal pathway is associated with the progression of several pathologies, including neurodegenerative diseases [12]. Indeed, there is substantial evidence that autophagy is dysregulated in pathologies such as AD, HD, PD, and TSEs [13-17], contributing to the accumulation of misfolded protein aggregates and neuronal death. Since drug-induced stimulation of autophagy flux produces anti-prion effects [18-25], autophagy is now emerging as a neuronal defense response in controlling prion infection, facilitating the clearance of aggregation-prone proteins and conferring a neuroprotective effect against prion-mediated neurotoxicity.

The presence of autophagic vacuoles is well demonstrated in experimentally induced scrapie, in the natural human prion disease, and in other experimental models of TSE [26-30]. Using sheep naturally affected with classical scrapie as a natural TSE animal model, we described earlier an altered, brain region-dependent regulation of autophagy [31]. The downregulation of transcripts for two autophagyrelated genes, ATG5 and ATG9, and the accumulation of p62 but not LC3, suggested an extensive decrease of the autophagic activity in injured CNS. However, the increased expression of LC3 and p62 proteins in some specific neuronal populations indicated that autophagy was still active in less lesioned areas. Our subsequent study in induced ovine atypical scrapie confirmed the impairment of autophagy in highly prion-affected brain areas [32]. However, whether autophagic dysregulation is a cause or a consequence of prion-induced toxicity is still unknown.

Besides naturally infected animals, experimentally induced murine models are extremely useful for carefully monitoring the disease progression and, especially, for studies of early stages of the pathology. The aim of the present study was to validate if the $\operatorname{Tg} 338$ mice that overexpress the ovine VRQ $\left(\mathrm{V}_{136} \mathrm{R}_{154} \mathrm{H}_{171}\right)$ allele for the PRNP (prion protein) gene related to high susceptibility to scrapie [33] can serve as a reliable model to mimic autophagy changes observed in the natural model. For this purpose, autophagy regulation was investigated through the immunohistochemical determination of LC3-B and p62 in Tg338 mice infected with ovine scrapie and the relationship between autophagy markers and scrapie-related lesions was analyzed. Moreover, as the use of murine models may facilitate the study of disease-associated molecular mechanisms, we investigated the regulation of selected messenger RNAs (mRNAs) and non-coding RNAs involved in autophagic machinery in mesencephalon (Mes) and cervical spinal cord (SC) of $\mathrm{Tg} 338$ mice at different stages of the disease.

\section{Materials and methods}

\section{Animals and sample processing}

Mouse bioassay was performed in $\operatorname{Tg} 338$ mice, a transgenic murine model that express in the brain the VRQ allele of the ovine PRNP gene at levels 8- to 10-fold higher than those detected in sheep brains [33]. All inocula were prepared manually in sterile saline as $10 \%$ CNS homogenates and were cultivated in blood agar plates to test for the absence of bacterial contamination. Intracerebral inoculations were performed under gaseous anesthesia using $50-\mu$ l syringes and 25-gauge hypodermic needles inserted into the right frontal lobe, delivering $20 \mu \mathrm{l}$ of the brain homogenate to each animal. To reduce post-inoculation pain, a subcutaneous injection of buprenorphine $(0.3 \mathrm{mg} / \mathrm{kg})$ was administered to each mouse before recovery to consciousness.

Two groups of six mice of 49 and 53 days of age were inoculated using the same Tg338-adapted classical scrapie source. This inoculum was prepared as a pool of cervical SC tissues from clinically infected $\operatorname{Tg} 338$ mice previously inoculated with CNS material from a naturally scrapieaffected ARQ/ARQ sheep. In order to study autophagy at the preclinical and clinical stages of the prion disease, one of these groups (preclinical) was inoculated with a delay of 55 days with respect to the first one (clinical). Two other groups of six mice were intracerebrally inoculated at equivalent times with an inoculum obtained from the CNS of non-inoculated $\operatorname{Tg} 338$ mice. They were selected as agematched controls for the preclinical and clinical groups of mice. The experimental groups are listed in Supplementary Table 1.

After inoculation, the mice were housed in filtered cages and their clinical status was monitored three times a week. Mice of the clinical group were euthanized when progression of the disease was evident upon detection of clinical signs of terminal prion disease. Mice from the preclinical and control groups were euthanized when the last mouse from the clinical group succumbed to the disease. After euthanasia, brains were harvested and divided sagittally. 
One hemisphere was immediately frozen in dry ice and conserved at $-80{ }^{\circ} \mathrm{C}$ and the other one was fixed by immersion in $10 \%$ formalin for up to $48 \mathrm{~h}$. From each mouse a section of Mes and a caudal section of the brain, including cervical SC, were preserved in RNAlater for gene expression study.

\section{Neuropathological evaluation}

$\operatorname{Tg} 338$ brains fixed in formalin were embedded in paraffin wax, cut into 4- $\mu$ m-thick sections, and mounted on glass slides for morphological evaluation using hematoxylin and eosin (HE) staining. Analysis of $\mathrm{PrP}^{\mathrm{Sc}}$ deposition was performed using the paraffin-embedded tissue (PET) blot method as previously described [34]. Briefly, paraffinembedded sections $(4 \mu \mathrm{m})$ were collected onto nitrocellulose membranes $(0.45 \mu \mathrm{m}$ pore size; Bio-Rad $)$ and digested with $250 \mu \mathrm{g} / \mathrm{ml}$ of proteinase $\mathrm{K}$ for $2 \mathrm{~h}$ at $56^{\circ} \mathrm{C}$. Membraneattached proteins were denatured with $3 \mathrm{M}$ guanidine thiocyanate (Sigma-Aldrich) and $\operatorname{PrP}^{\mathrm{Sc}}$ was detected using Sha31 mouse monoclonal antibody (1:8000 dilution for $1 \mathrm{~h}$; SPI-Bio). Sections were then incubated with an alkaline phosphatase-coupled goat anti-mouse antibody (1:500 dilution for $1 \mathrm{~h}$; Dako) and enzymatic activity was visualized using NBT/BCIP chromogen (Sigma-Aldrich).

\section{Immunohistochemical analysis of autophagy markers}

Paraffin-embedded CNS tissue sections from Tg338 mice were studied by immunohistochemistry (IHC) to evaluate the brain expression and distribution of LC3-B and p62 autophagy-related proteins. After deparaffination and rehydration, sections were pre-treated by heat-induced epitope retrieval for $20 \mathrm{~min}$ at $96^{\circ} \mathrm{C}$ in a PTLink (Dako), using TrisEDTA buffer (pH 9.0). Endogenous peroxidase activity was then blocked and samples were incubated for $1 \mathrm{~h}$ at room temperature with the following primary antibodies: mouse monoclonal anti-MAP-LC3 $\beta$ (1:200 dilution; G-2, sc271625; Santa Cruz Biotechnology) and rabbit polyclonal anti-p62 (1:200 dilution; PW9860; Enzo Life Sciences). Omission of the primary antibody served as a background control for nonspecific binding of the secondary antibody. Subsequently, the enzyme-conjugated polymer EnVision (Dako EnVision anti-mouse for MAP-LC3 $\beta$; Dako EnVision anti-rabbit for p62) was used as visualization system, followed by an incubation with diaminobenzidine chromogen (Dako).

\section{Quantification of gene expression}

Five genes, described to be involved in autophagic machinery, were selected for the analysis of their expression profile in Mes and cervical SC of $\mathrm{Tg} 338$ mice: Atg5 (autophagy-related 5), $L c 3, F b x w 7$ (F-box and WD repeat domain containing 7), p62, and Gas5 (growth arrest-specific 5). Supplementary Table 2 shows TaqMan assays (Thermo Fisher Scientific) used for the amplification of the genes of interest. Total RNA was isolated from RNAlater-conserved Mes and SC, using the Direct-Zol ${ }^{\mathrm{TM}}$ RNA kit (Zymo Research). Retrotranscription was performed from $200 \mathrm{ng}$ of total RNA using qScript ${ }^{\mathrm{TM}}$ cDNA Supermix (Quanta Biosciences ${ }^{\mathrm{TM}}$ ), according to the manufacturer's instructions. Resulting complementary DNA was diluted 1:5 in water and gene expression was quantified by quantitative real-time PCR using the TaqMan universal PCR master mix assays (Thermo Fisher Scientific) in a StepOne Plus RealTime PCR instrument (Applied Biosystems). All reactions were run in triplicate and universal amplification conditions were used. Expression levels of autophagy-related genes were normalized with Sdha housekeeping gene (Supplementary Table 2).

\section{Data analysis}

Histopathological lesions (i.e., spongiform changes), and LC3-B and p62 immunolabelling, were evaluated in each brain section under a Zeiss Axioskop 40 optical microscope, whereas the intensity and distribution of $\mathrm{PrP}^{\mathrm{Sc}}$ deposition were analyzed with a Zeiss Stemi DV4 stereomicroscope. A semi-quantitative approach was used to obtain comparable data from the different lesions and autophagy markers in a total of nine brain regions described by Fraser and Dickinson [35]: frontal cortex, septal area, thalamic cortex (Tc), hippocampus $(\mathrm{Hc})$, thalamus $(\mathrm{T})$, hypothalamus $(\mathrm{Ht})$, Mes, cerebellum $(\mathrm{Cbl})$, and medulla oblongata (Mo). Each area was investigated globally as a region for the scoring, except for $\mathrm{Cbl}$, whose layers [molecular layer (Ml), Purkinje layer $(\mathrm{Pl})$, granular layer, white matter $(\mathrm{Wm})$, and deep cerebellar nuclei (DCN)] were analyzed separately in order to obtain a more detailed description. Brain regions studied were scored blindly and separately for the degree of spongiosis, $\operatorname{Pr} \mathrm{P}^{\mathrm{Sc}}$ deposition and autophagy proteins on a scale ranging from 0 (absence of spongiosis or immunolabelling) to 5 (maximum intensity of neuropathological lesions or immunolabelling).

Significant differences of spongiform changes, $\mathrm{PrP}^{\mathrm{Sc}}$ deposition profiles, and LC3-B and p62 protein distribution between the experimental mouse groups were evaluated using the non-parametric Mann-Whitney $U$ test. Relative gene expression quantification was determined using the $2^{-\Delta \Delta C t}$ method and results were analyzed with the Student's $t$ test. Previously, normal distribution of data was confirmed using the Shapiro-Wilk test. Correlations between autophagy proteins immunostaining and histopathological lesions were determined using the non-parametric Spearman's rank correlation coefficient (rho, $\rho$ ). We used IBM $^{\bullet}$ SPSS $^{\circ}$ 
statistics 22 software for all data analysis, and GraphPad Prism version 6.0 to perform the graphs. In all tests, the results were considered significant at $P<0.05$.

\section{Results}

\section{Clinical signs of murine prion disease in Tg338 mice inoculated with ovine scrapie}

The mouse-adapted classical scrapie inoculum used in the present study transmitted with $100 \%$ attack rate in the group of $\operatorname{Tg} 338$ mice intended for autophagy evaluation in the clinical stage of the disease (clinical group). These mice were euthanized with terminal clinical signs compatible with TSE at $\sim 170$ days post inoculation (dpi) and aged $\sim 219$ days (Supplementary Table 1). Early clinical signs included mild dorsal kyphosis, tail rigidity, and poor hair coat. Subsequently, mice developed clinical signs of terminal disease, including severe ataxia, blepharitis, weight loss, and hunched stance. In contrast, mice inoculated with a delay of 55 days were euthanized at $136 \mathrm{dpi}$ without clinical signs (preclinical group). Control mice inoculated with healthy $\operatorname{Tg} 338$ brain material and euthanized after 136 (age-matched controls for preclinical mice) or 191 dpi (age-matched controls for clinical mice) did not display signs of disease.

\section{Histopathological profiles and PrP ${ }^{\mathrm{Sc}}$ deposition patterns in the CNS of scrapie-inoculated Tg338 mice are similar to those described in classical scrapie sheep}

Clinical $\operatorname{Tg} 338$ mice showed a significant increment of spongiform changes in all brain areas evaluated compared to their control group, except for $\mathrm{Cbl}$ (Supplementary Fig. 1a). In these animals, T, Ht, Mes, and Mo displayed the most severe spongiform lesions, while lesions were moderate in Hc and cerebral cortex and minimal in Cbl. Preclinical and control groups displayed minimal spongiform lesion scores. In addition, lesion scores for clinically affected mice were also significantly higher than those for the preclinical group in T, Ht, Mes, and Mo. No significant changes were detected when comparing mice at the preclinical stage to their age-matched control group, nor between mock-treated control groups.

All Tg338 mice that had developed clinical signs were positive for $\operatorname{PrP}^{\mathrm{Sc}}$ accumulation in the brain (see representative PET blot in Supplementary Fig. 1b). In this group of animals, $\operatorname{PrP}^{\mathrm{Sc}}$ deposition was especially remarkable in Mes, $\mathrm{Ht}$, and Mo. However, Mo showed large variability in $\operatorname{PrP}^{\mathrm{Sc}}$ deposition intensity between clinical animals, leading to a high deviation in the semi-quantitative scoring. No $\operatorname{PrP}^{\mathrm{Sc}}$ deposition was detected in mice at the preclinical stage.
LC3-B protein is downregulated and p62 is upregulated in some highly affected brain areas of scrapie-infected Tg338 mice at the clinical stage

Immunohistochemical determination of LC3-B and p62 in $\operatorname{Tg} 338$ mice revealed differential regulation of autophagic activity in those inoculated with ovine scrapie. Infected $\operatorname{Tg} 388$ mice at the clinical stage obtained the lowest scores for LC3-B in all CNS areas analyzed (Fig. 1a), this downregulation being statistically significant in $\mathrm{T}(P=0.047), \mathrm{Ht}$ $(P=0.028)$, Mes $(P=0.028), \mathrm{Pl}(P=0.015)$, and Mo $(P=$ 0.023 ) compared to their controls (Fig. 1b). Moreover, the three first aforementioned areas and DCN also showed a significant decrease $(P=0.038$ for $\mathrm{T}, 0.033$ for $\mathrm{Ht}, 0.023$ for Mes, and 0.028 for DCN, respectively) in clinical animals with respect to scrapie-inoculated mice at the preclinical stage of the disease. Preclinical scrapie mice displayed similar LC3-B scores than their age-matched controls (Supplementary Fig. 2a). In preclinical mice and the clinical control group, LC3-B immunohistochemical pattern was characterized by an intense and uniform intraneuronal immunolabelling affecting numerous cells, and by an abundant punctiform diffuse staining in the neuropil (Fig. 2). However, the clinical mice displayed a lower number of immunopositive cells and a slight neuropil immunolabelling.

In contrast to LC3-B, p62 immunostaining was clearly stronger in brains from clinically affected $\operatorname{Tg} 338$ mice (Fig. 1c). These animals seemed to accumulate the protein in all CNS areas analyzed, the increment being statistically significant in $\mathrm{Ht}(P=0.019), \mathrm{Ml}(P=0.019)$, and $\mathrm{Pl}(P=$ 0.038 ) when compared to the preclinical mice, and in $\mathrm{Ht}$ $(P=0.045)$ when compared to their control group (Fig. 1d). In addition, a tendency to upregulation was detected in Tc $(P=0.088), \mathrm{Pl}(P=0.095)$, and $\mathrm{Wm}(P=0.077)$ in clinical mice with respect to the control group. Similar to LC3-B, no significant differences were detected between preclinical mice and their control group (Supplementary Fig. 2b), nor between control groups. Both neuronal and glial cells displayed a strong intracellular p62 immunolabelling in the clinical scrapie group, which also presented a remarkable granular staining of the neuropil (Fig. 3). Although presenting the same pattern, both the number of stained cells and the intensity of immunolabelling were lower in the preclinical and the control group.

\section{Dysregulation of LC3-B and p62 is associated to prion-related neuropathology}

To identify the relationship between scrapie-related lesions and autophagy, Spearman's $\rho$ correlation was calculated between immunohistochemical and histopathological scores. Table 1 shows correlation values and their statistical significance. Surprisingly, although LC3-B expression 

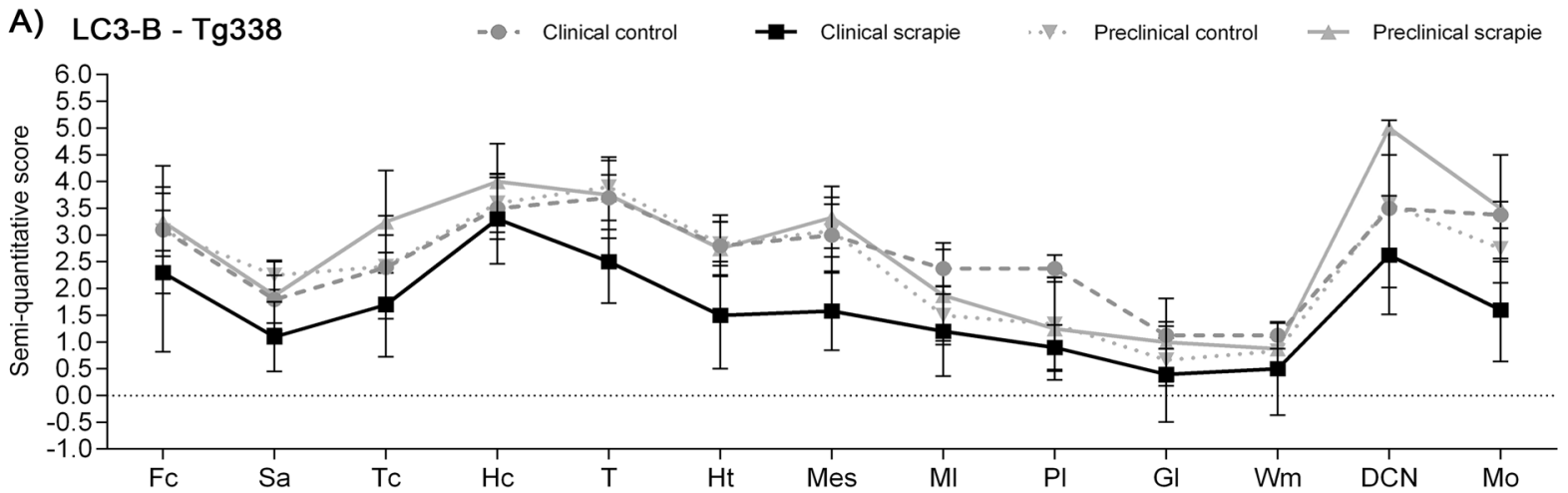

B) LC3-B - Tg338 (differences between groups)

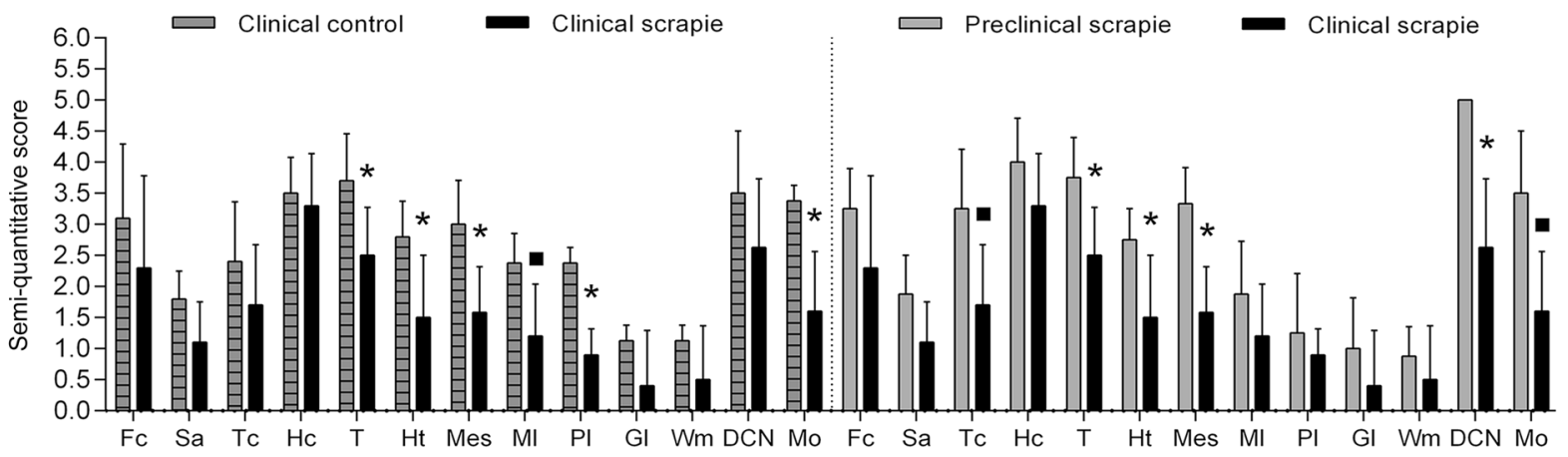

C) $\mathrm{p} 62-\operatorname{Tg} 338$

- C Clinical control

$\rightarrow$ Clinical scrapie

$\nabla$ Preclinical control $\leftarrow$ Preclinical scrapie

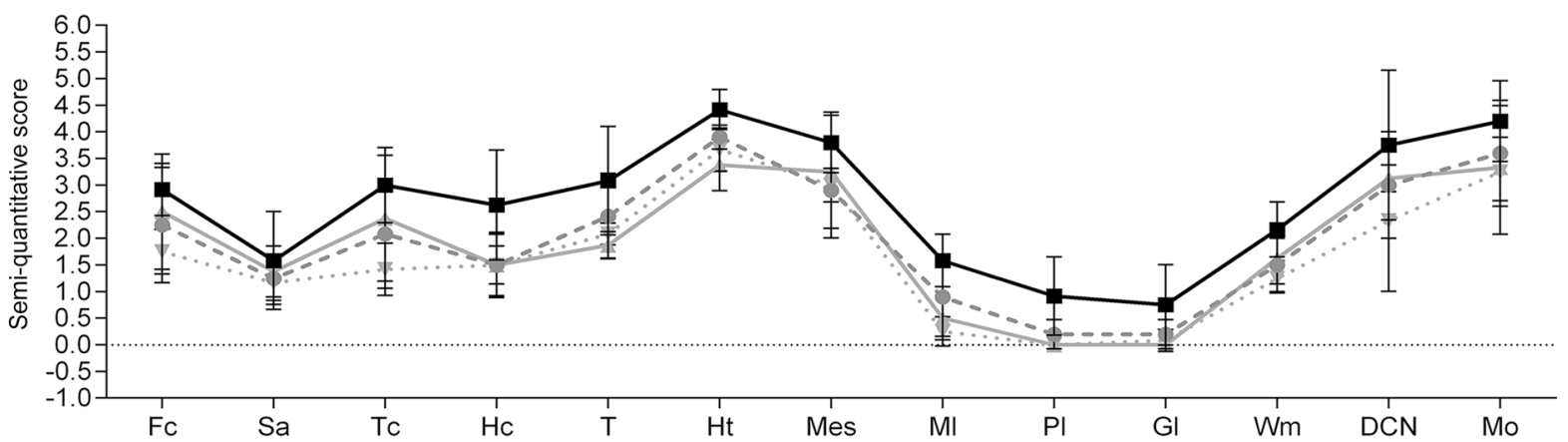

D) p62 - Tg338 (differences between groups)

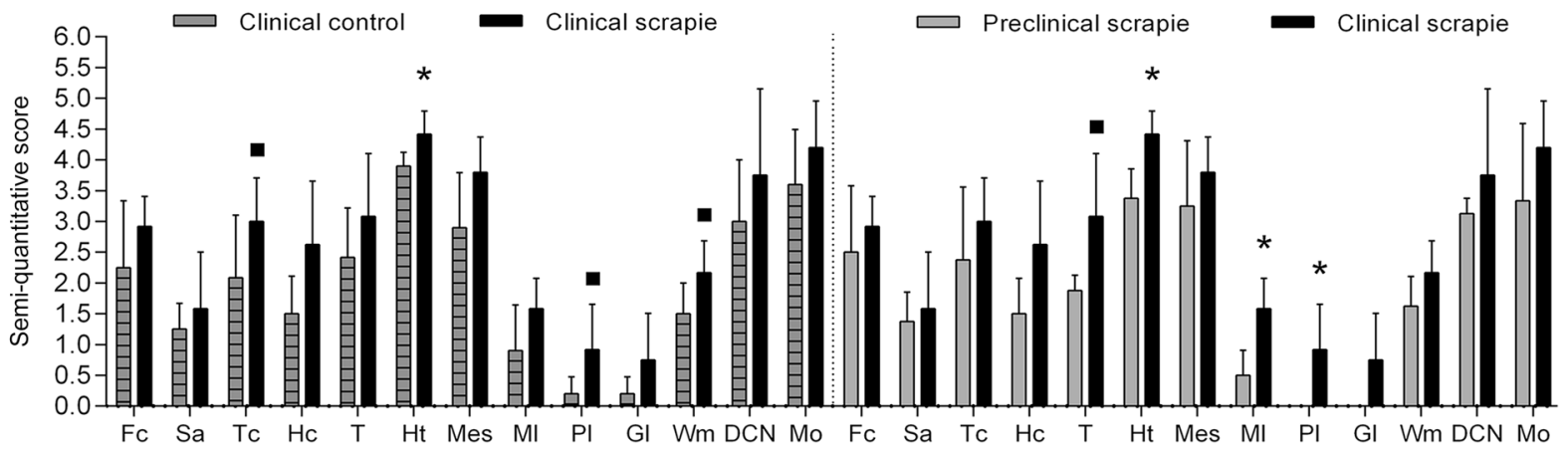

decreased in clinical mice, LC3-B immunostaining scores displayed a slight but statistically significant positive correlation with spongiform lesions in the total set of animals
( $\rho=0.242, P<0.001)$. However, this correlation seemed to be related with the preclinical stage of the disease where correlations were significant in both the total set $(\rho=0.462$, 
Fig. 1 Semi-quantitative scoring of LC3-B and p62 in the CNS of Tg338 scrapie-infected mice. Figure shows a, c global graphs and b, d comparative graphs of the clinical group (black bars) with preclinical (gray bars) and control group (gray striped bars). Comparative graphs between preclinical and their control group, and between control groups, are not shown because no significant differences were detected. Score values (from 0: negative, to 5: staining present at its maximum intensity) evaluated in frontal cortex $(\mathrm{Fc})$, septal area $(\mathrm{Sa})$, thalamic cortex $(\mathrm{Tc})$, hippocampus $(\mathrm{Hc})$, thalamus $(\mathrm{T})$, hypothalamus (Ht), mesencephalon (Mes), cerebellum [which includes molecular layer (Ml), Purkinje layer $(\mathrm{Pl})$, granular layer $(\mathrm{Gl})$, white matter $(\mathrm{Wm})$, and deep cerebellar nuclei (DCN)] and medulla oblongata (Mo). The differences between the experimental groups were determined using the Mann-Whitney $U$ test $(" P<0.1$ and $* P<0.05)$

$P<0.001)$ and only when the scrapie-inoculated animals were analyzed $(\rho=0.426, P=0.003)$. There was no correlation between the scrapie lesions and LC3-B in the clinical stage of the disease. In contrast, p62 expression was strong and positively correlated with spongiform lesions in all groups of individuals ( $\rho>0.68, P<0.001$ for all cases). This correlation suggests a relationship between the accumulation of p62 and the course of the disease and degree of spongiform lesion. Intensity of prion deposition determined by PET blot negatively correlated with LC3-B immunostaining in the total set of scrapie-inoculated animals $(\rho=$ $-0.473, P<0.001)$, but not when the preclinical or clinical groups were separated. On the contrary, p62 immunostaining positively correlated with prion deposition in the total set of scrapie animals $(\rho=0.300, P=0.009)$, but not in the individual groups. These correlations confirm the decrease of LC3-B and increase of p62 in clinical animals, but the observed distribution patterns do not seem to be related with the degree of $\mathrm{PrP}^{\mathrm{Sc}}$ deposition.

\section{mRNA expression of Gas5, Atg5, and Fbxw7 is downregulated in the CNS of scrapie-infected Tg338 mice at the clinical stage}

Murine models may provide valuable tools for analyzing the molecular mechanisms underlying the pathological processes in TSEs. In this work, transcripts from a selected group of genes encoding factors involved in autophagy were quantified in Mes and SC of the four $\mathrm{Tg} 338$ mouse groups. Mes is one of the most prion-affected brain areas in this murine model (Supplementary Fig. 1) and SC is adjacent to the Mo, and therefore they share similar levels of scrapie neuropathology in sheep [36]. As shown in Fig. 4a, Gas5 displayed a significant downregulation $(P=0.03)$ in Mes of the clinical mice. In addition, a significant decrease of the expression of $\operatorname{Atg} 5(P=0.04)$ and Fbxw7 $(P=0.03)$ was detected in SC of the same group (Fig. 4b). Significant changes were not detected at the preclinical stage of the disease.

\section{Discussion}

Prion diseases, progressive and fatal neurodegenerative disorders of humans and animals, are characterized by the accumulation of misfolded and aggregated $\operatorname{PrP}^{\mathrm{Sc}}$ in the CNS, resulting in spongiform degeneration. Although autophagic vacuoles appear in several models of TSEs [2630], little is known about how this process is involved during the course of the disease. Consequently, the relationship between autophagy and prion-related pathology is still open to debate. In a previous work, we investigated the dynamics of autophagy in the CNS of sheep naturally affected with classical scrapie [31]. In this natural model, we described a possible impairment of autophagy in some of the most highly affected brain regions, whereas mechanisms compatible with induction of autophagy were observed in less lesioned areas, suggesting a combination of anti-prion and prion-promoting effects throughout the brain during the clinical stage of the disease. The impairment of autophagy in highly prion-affected brain areas was subsequently confirmed in sheep experimentally infected with atypical scrapie [32]. Murine models are widely used to perform in vivo assays and analyze the molecular mechanisms of the disease at different stages of the pathological process. In the present work, using a combination of immunohistochemical staining and real-time PCR quantification of markers involved in autophagy, we further investigated the role of autophagy in the $\operatorname{Tg} 338$ mouse model that overexpresses the most sensitive allele (VRQ) to classical scrapie infection of the ovine PRNP gene [33], in order to evaluate its potential as a model for the natural TSE.

We first confirmed that clinical $\operatorname{Tg} 338$ mice display consistent similarities in neuropathological lesion profiles and $\mathrm{PrP}^{\mathrm{Sc}}$ deposition patterns in PET blots to those observed in classical scrapie sheep [31]. The scrapie-inoculated Tg338 transgenic mice showed a minimal effect on $\mathrm{Cbl}$ and highest in $\mathrm{T}$ and brainstem. The presence of some spongiform lesions in the preclinical and control $\mathrm{Tg} 338$ mice could be related to the transgenic overexpression of $\operatorname{PrP}^{\mathrm{c}}$, as also described by Westaway et al. [37].

As comparative analysis about LC3-B and p62 accumulation is necessary for the evaluation of autophagic activity [38, 39], we then investigated the dynamics of autophagy during prion pathogenesis in scrapie-infected Tg338 mice by estimating the expression levels of these proteins by IHC. Immunostaining assays are equally efficient as Western blots to observe even slight changes of p62 aggregation and, in addition, they can also provide further information, such as the number, size, and intracellular distribution of the aggregates [40].

The protein p62 is a common constituent of neuronal and glial inclusions in various neurodegenerative diseases, such 


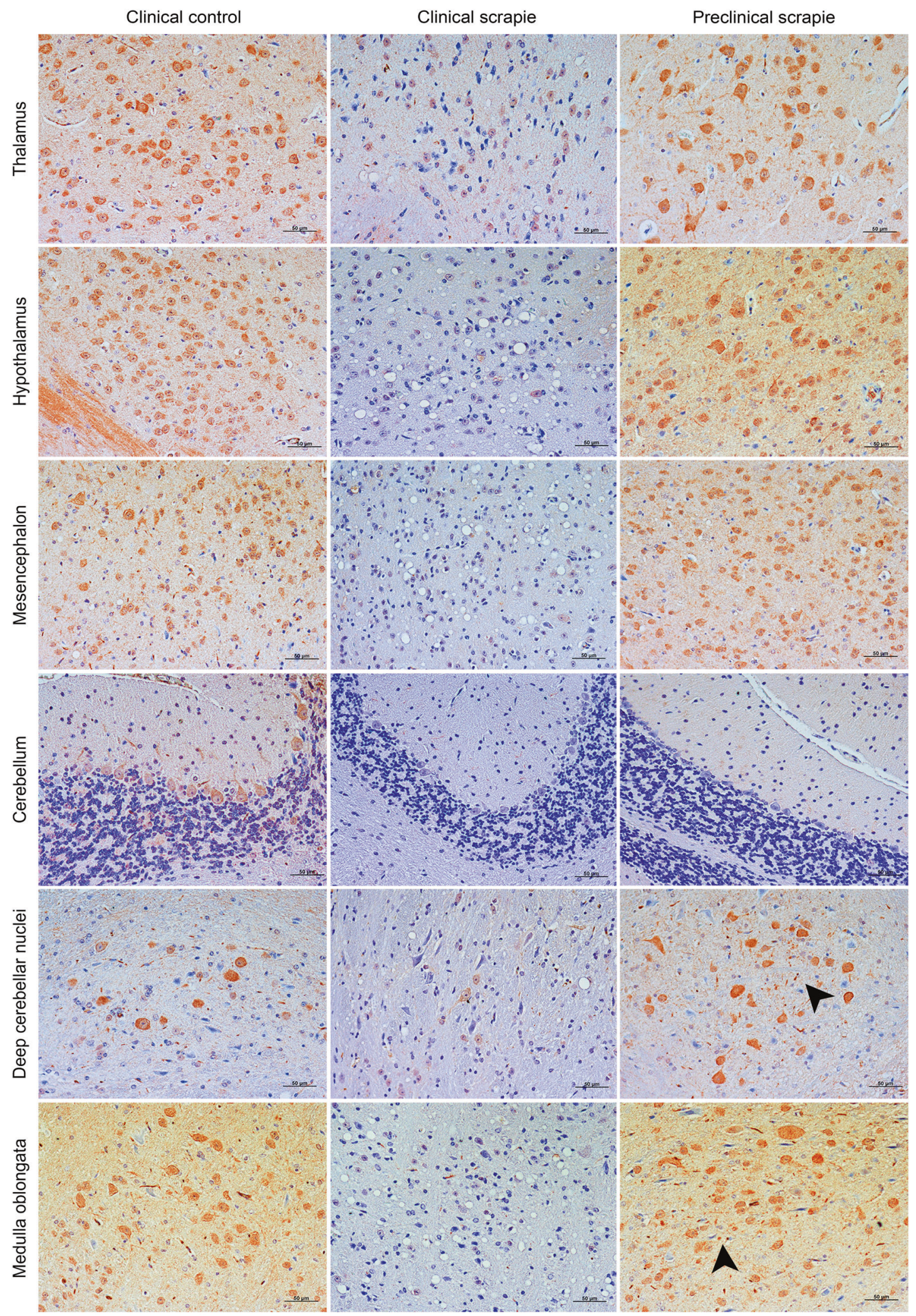

as $\mathrm{AD}$ and PD [41-43]. Furthermore, it is induced as a response to the expression of mutant huntingtin [44]. Our study did not reveal differential p62 regulation at the transcript level, but the amount of this protein increased significantly in $\mathrm{Ht}$ and some cerebellar layers of scrapieinfected Tg338 mice at the clinical stage. These animals 
Fig. 2 Immunostaining patterns of LC3-B in different CNS areas of Tg338 scrapie-infected mice. Figure shows representative images of LC3-B immunostaining in the clinical (middle), preclinical (right), and control group (left) $(50 \mu \mathrm{m})$. Clinical scrapie-infected mice displayed a lower number of immunopositive cells and a slight neuropil immunolabelling in thalamus, hypothalamus, and mesencephalon compared to the preclinical and control groups. In cerebellum, significant downregulation of LC3-B was observed in Purkinje layer in clinical mice when compared to their control group, and in the deep cerebellar nuclei when compared to the preclinical group. In medulla oblongata, LC3-B was significantly decreased in clinical mice compared to their control group, but also a tendency towards downregulation was observed with respect to the preclinical scrapie mice. Note the abundant punctiform staining in the neuropil in deep cerebellar nuclei and medulla oblongata (arrowheads)

also displayed p62 immunolabelling in glial cells. In agreement with our results, levels of p62 protein increase in prion-infected cell cultures and in brains of scrapie $263 \mathrm{~K}$ infected hamsters and scrapie 22L-infected mice [45]. In the latter study, LC3-II protein was also upregulated in 22Linoculated mice, suggesting that activation of p62 could promote the clearance of $\mathrm{PrP}^{\mathrm{Sc}}$ through the involvement of autophagic degradation. In contrast, scrapie-infected mice at the clinical stage in our study displayed a significant downregulation of LC3-B at the protein level in the most prion-affected areas (i.e., T, Ht, Mes, and Mo) and in some cerebellar layers, such as $\mathrm{Pl}$ and DCN. The interpretation of the results based on the detection of autophagy markers is not straightforward. Whereas some studies relate the inhibition of autophagy with an increase of LC3-II levels [46], many others consider that the increment of LC3-II reflects an induction of autophagy [45, 47, 48]. This increment is accompanied by a downregulation of p62 in the terminal stage of 263K-infected hamsters and in human genetic prion diseases [48] and an upregulation of p62 in neuron cells treated with amino-terminally truncated prion protein (PrP90-231) [47]. However, the latter study suggested that PrP peptide stimulates autophagic flux, but leads progressively to the accumulation of autophagolysosomes with impaired resolution ability, because it is widely accepted that autophagic flux inhibition leads to p62 accumulation [49]. Consequently, the increment of p62 and the downregulation of LC3-B in our experimental model does not seem to reflect an enhancement of autophagic activity but an impairment of it, which would promote the progression of the disease during the terminal stages.

Our molecular results regarding the expression of autophagy-related genes are also in agreement with the possible inhibition of the autophagy pathway. The rationale for selecting the genes for the expression studies was that they are positive regulators of autophagy through their action on formation of autophagic vesicles directly (Atg5) [50], indirectly through microRNA sponging (Gas5) [51], or through inhibition of mammalian target of rapamycin complex (Fbxw7) [52]. Here, we have described a downregulation of Atg5 in SC of clinical stage Tg338 mice, but not in the preclinical group. Neuron deletion of Atg5 in mice causes accumulation of ubiquitinated proteins, damaged organelles, and autophagy-specific substrates such as p62 [53]. In addition, downregulation of mRNA levels of beclin-1 and Atg5 in brains of scrapie-infected wild-type

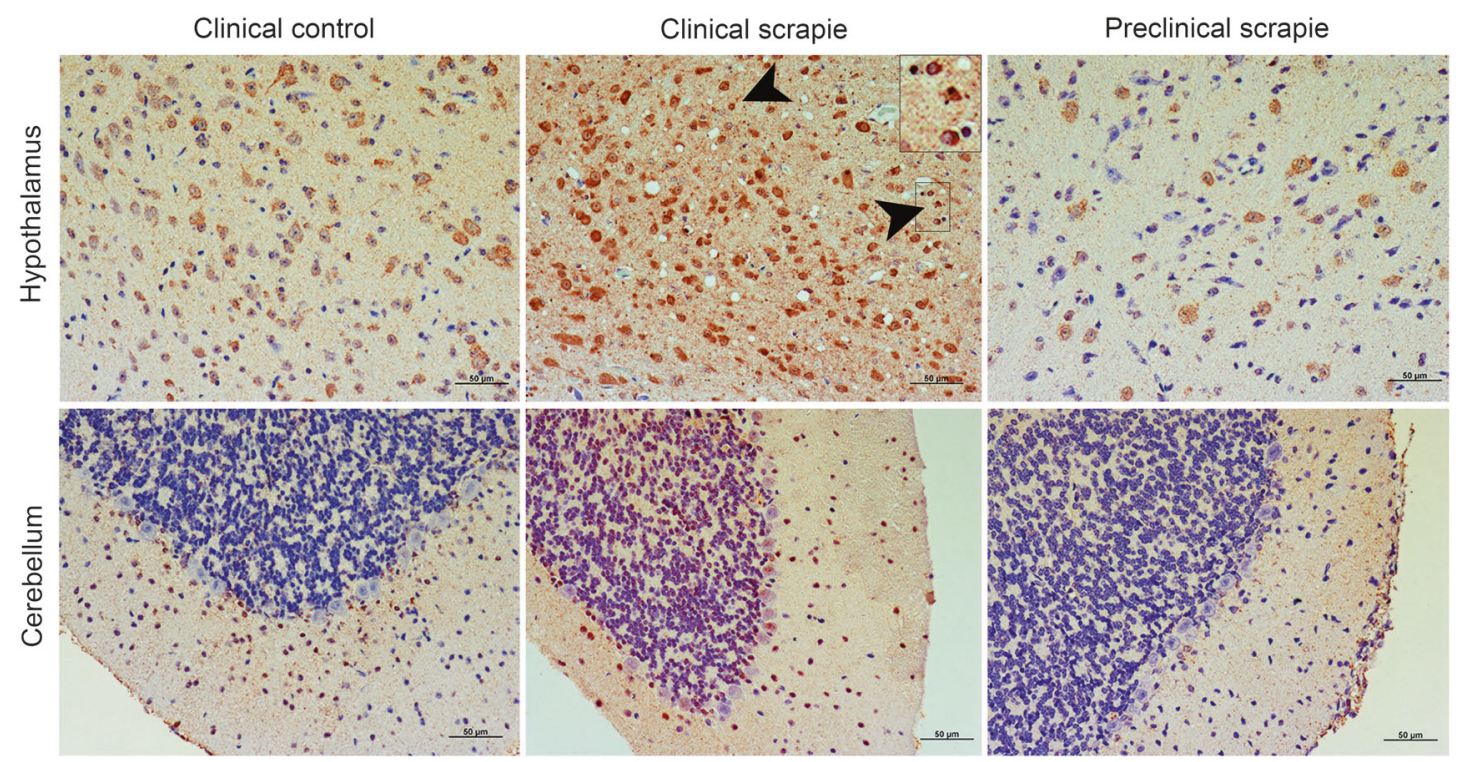

Fig. 3 Immunostaining patterns of p62 in different CNS areas of Tg338 scrapie-infected mice. Figure shows representative images of p62 immunostaining in the clinical (middle), preclinical (right), and control group (left) $(50 \mu \mathrm{m})$. p62 Immunostaining was significantly stronger in hypothalamus in clinical mice compared to the preclinical and control groups, and in molecular layer and Purkinje layer of cerebellum compared to the preclinical group. Notice the glial cell immunolabelling (arrowheads and detail) 
Table 1 Spearman's correlation values between scores of autophagy markers (LC3-B and p62) and scrapie-related histopathological lesions (spongiform changes and $\mathrm{PrP}^{\mathrm{Sc}}$ deposition $)^{\mathrm{a}}$

\begin{tabular}{|c|c|c|c|c|c|c|}
\hline & \multicolumn{2}{|c|}{ Preclinical stage } & \multicolumn{2}{|c|}{ Clinical stage } & \multirow[t]{2}{*}{ Total set } & \multirow{2}{*}{$\begin{array}{l}\text { Scrapie clinical + } \\
\text { preclinical }\end{array}$} \\
\hline & $\begin{array}{l}\text { Control + } \\
\text { scrapie }\end{array}$ & Scrapie & $\begin{array}{l}\text { Control + } \\
\text { scrapie }\end{array}$ & Scrapie & & \\
\hline \multicolumn{7}{|l|}{ LC3-B } \\
\hline Spongiosis & $0.462 * * *$ & $0.426 * * *$ & $0.09^{\text {n.s. }}$ & $0.271^{\text {n.s. }}$ & $0.242 * * *$ & $0.180^{\text {n.s. }}$ \\
\hline $\mathrm{PrP}^{\mathrm{Sc}}$ & - & $-0.203^{\text {n.s. }}$ & - & $-0.054^{\text {n.s. }}$ & - & $-0.473 * * *$ \\
\hline \multicolumn{7}{|l|}{ p62 } \\
\hline Spongiosis & $0.720 * * *$ & $0.760 * * *$ & $0.682 * * *$ & $0.768 * * *$ & $0.700 * * *$ & $0.766^{* * *}$ \\
\hline $\operatorname{PrP}^{\mathrm{Sc}}$ & - & $-0.040^{\text {n.s. }}$ & - & $0.235^{\text {n.s. }}$ & - & $0.300 * *$ \\
\hline
\end{tabular}

Correlations were estimated using the full set of data obtained in all tissues

n.s. No statistically significant value

Spearman's correlation, $* * P<0.01$ and $* * * P<0.001$

${ }^{a}$ In the preclinical and clinical stage, in the total set of animals and only in scrapie-inoculated mice
A) Gene expression in mesencephalon

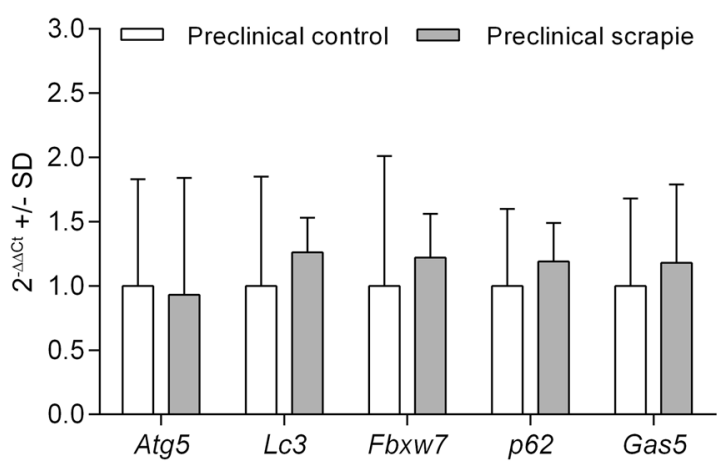

B) Gene expression in cervical spinal cord

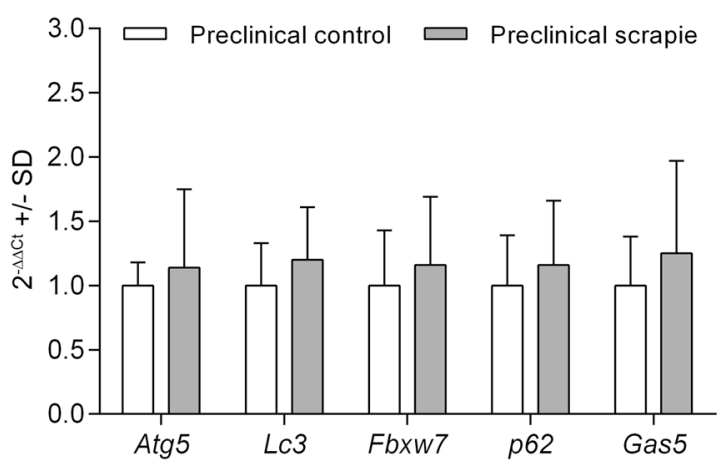

Fig. 4 Gene expression profiles of Atg5, Lc3, Fbxw7, p62, and Gas5 in Tg338 scrapie-infected mice. Graphics show mRNA expression profiles in a mesencephalon and $\mathbf{b}$ cervical spinal cord, in preclinical (left, gray bars) and clinical (right, black bars) Tg338 scrapie-infected mice.

mice has been associated with an impairment of autophagy [17]. Our results also mimic the negative regulation of ATG5 in $\mathrm{T}$ observed in clinically scrapie-infected sheep [31].

In the same animals and tissue, also $F b x w 7$ mRNA was downregulated. Knockdown of $F b x w 7$ inhibits autophagic
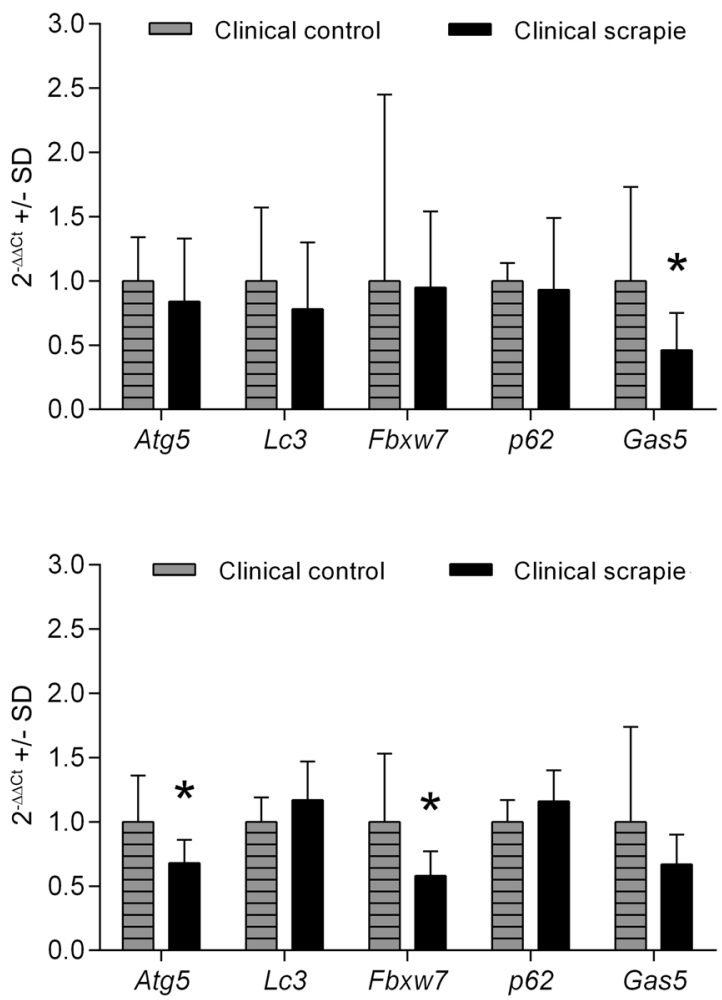

Relative expression levels are expressed as mean \pm standard deviation (SD). Results were normalized using the expression of Sdha housekeeping gene. The expression values were determined using the $2^{-\Delta \Delta \mathrm{Ct}}$ method and results were analyzed with the Student's $t$ test $(* P<0.05)$

flux and increases $\operatorname{PrP}^{\mathrm{Sc}}$ accumulation in SMB-S15 cells infected with brains of scrapie agent 263K [52]. Moreover, the expression of the long non-coding RNA Gas 5 decreased in Mes of scrapie-infected mice at the clinical stage. Alterations in the expression of Gas 5 have been reported in a range of animal pathological models [54], but it has not 
been investigated in prion diseases before. Depletion of Gas 5 results in decreased autophagy in non-small-cell lung carcinoma cells [55] and, in epithelial cells, knockdown of Gas5 suppresses the expression of LC3-II, ATG3, and ATG5-ATG12 complex formation, whereas p62 levels are promoted [56]. Although further studies are needed to completely define the role of some of these regulatory factors in the specific conditions of prion infection, the above-mentioned findings on autophagy genes together with the decrease of LC3-B protein and the accumulation of p62 in the clinical mice group suggest that autophagic machinery is impaired during the late stage of prion infection.

To conclude, the specific role of autophagy in prion diseases is still controversial. However, there is good evidence that autophagic process decreases in misfolded protein-mediated neurodegenerative diseases such as $\mathrm{AD}$ [57] or HD [58]. The present data suggest that autophagy is part of the pathological process and that modulation of autophagic capacity could play an essential role in the pathogenesis of prion diseases. The negative regulation of autophagy-related genes and the upregulation and downregulation of p62 and LC3-B proteins, respectively, observed in clinical scrapie-infected mice, but not in the preclinical group, likely reflect an impairment of the autophagic pathway that seems to take place at the last stage of the disease. Moreover, the absence of changes in autophagy regulation at the preclinical stage suggests the impairment of autophagy at the clinical stage is not the result of depletion or exhaustion of the autophagic machinery as suggested by our previous results in natural scrapie, where changes compatible with autophagy induction were observed in less affected CNS areas [31]. These discrepancies with the natural model may be related to the more aggressive disease development in the experimental transgenic model used and, therefore, the findings described here may not fully reflect the mechanisms underlying the natural disease. Although further studies involving late preclinical animals, different strains, and experimental models are needed to clarify these discrepancies, the alteration of autophagy during the clinical stage of the disease and the resemblance of prion neuropathology between both models demonstrate the suitability of $\mathrm{Tg} 338$ murine model to study the implication of autophagy in prion diseases.

Acknowledgements We would like to express our gratitude to Sonia Gómez and Daniel Romanos (University of Zaragoza) for technical assistance. OL-P and AO were supported by research grants from Gobierno de Aragón (C012/2014; C020/2014) co-financed by the European Social Fund. This work was partially funded by the Ministry of Economy and Competitiveness of the Government of Spain (Grant AGL2015-67945-P), the Istituto de Salud Carlos III (Grant PI17/ 00949) and Fondo Europeo de Desarrollo Regional (FEDER) "Una manera de hacer Europa", and the Government of Aragon (Reference Group A19-17R) cofinanced with FEDER 2014-2020 "Construyendo Europa desde Aragón".

\section{Compliance with ethical standards}

Conflict of interest The authors declare that they have no conflict of interest.

Ethics statement All experimental procedures performed in animals were approved by the Ethics Committee for Animal Experiments of the University of Zaragoza (Permit Number: PI40/15) and were carried out in compliance with the recommendations for the care and use of experimental animals established by Spanish law (R.D. 53/2013) and European Directive 2010/63/UE.

Publisher's note: Springer Nature remains neutral with regard to jurisdictional claims in published maps and institutional affiliations.

\section{References}

1. Prusiner SB. Novel proteinaceous infectious particles cause scrapie. Science. 1982;216:136-44.

2. Hadlow WJ, Eklund CM. Scrapie-a virus-induced chronic encephalopathy of sheep. Res Publ Assoc Res Nerv Ment Dis. 1968;44:281-306.

3. Wells GA, McGill IS. Recently described scrapie-like encephalopathies of animals: case definitions. Res Vet Sci. 1992;53:1-10.

4. Wood JL, McGill IS, Done SH, Bradley R. Neuropathology of scrapie: a study of the distribution patterns of brain lesions in 222 cases of natural scrapie in sheep, 1982-1991. Vet Rec. 1997;140:167-74.

5. Soto C. Unfolding the role of protein misfolding in neurodegenerative diseases. Nat Rev Neurosci. 2003;4:49-60.

6. Rubinsztein DC. The roles of intracellular protein-degradation pathways in neurodegeneration. Nature. 2006;443:780-6.

7. Jahreiss L, Menzies FM, Rubinsztein DC. The itinerary of autophagosomes: from peripheral formation to kiss-and-run fusion with lysosomes. Traffic. 2008;9:574-87.

8. Mizushima N, Yoshimori T. How to interpret LC3 immunoblotting. Autophagy. 2007;3:542-5.

9. Komatsu M, Waguri S, Koike M, Sou YS, Ueno T, Hara T, et al. Homeostatic levels of p62 control cytoplasmic inclusion body formation in autophagy-deficient mice. Cell. 2007;131:1149-63.

10. Bjorkoy G, Lamark T, Brech A, Outzen H, Perander M, Overvatn A, et al. p62/SQSTM1 forms protein aggregates degraded by autophagy and has a protective effect on huntingtin-induced cell death. J Cell Biol. 2005;171:603-14.

11. Wang QJ, Ding Y, Kohtz DS, Mizushima N, Cristea IM, Rout $\mathrm{MP}$, et al. Induction of autophagy in axonal dystrophy and degeneration. J Neurosci. 2006;26:8057-68.

12. Levine B, Kroemer G. Autophagy in the pathogenesis of disease. Cell. 2008;132:27-42.

13. Li L, Zhang X, Le W. Autophagy dysfunction in Alzheimer's disease. Neurodegener Dis. 2010;7:265-71.

14. Nixon RA. The role of autophagy in neurodegenerative disease. Nat Med. 2013;19:983-97.

15. Nixon RA, Wegiel J, Kumar A, Yu WH, Peterhoff C, Cataldo A, et al. Extensive involvement of autophagy in Alzheimer disease: an immuno-electron microscopy study. J Neuropathol Exp Neurol. 2005;64:113-22.

16. Suzuki K, Terry RD. Fine structural localization of acid phosphatase in senile plaques in Alzheimer's presenile dementia. Acta Neuropathol. 1967;8:276-84. 
17. Mok SW, Riemer C, Madela K, Hsu DK, Liu FT, Gultner S, et al. Role of galectin-3 in prion infections of the CNS. Biochem Biophys Res Commun. 2007;359:672-8.

18. Aguib Y, Heiseke A, Gilch S, Riemer C, Baier M, Schatzl HM, et al. Autophagy induction by trehalose counteracts cellular prion infection. Autophagy. 2009;5:361-9.

19. Bolognesi ML, Legname G. Approaches for discovering antiprion compounds: lessons learned and challenges ahead. Expert Opin Drug Discov. 2015;10:389-97.

20. Forloni G, Artuso V, Roiter I, Morbin M, Tagliavini F. Therapy in prion diseases. Curr Top Med Chem. 2013;13:2465-76.

21. Gilch S, Krammer C, Schatzl HM. Targeting prion proteins in neurodegenerative disease. Expert Opin Biol Ther. 2008;8:923-40.

22. Goold R, McKinnon C, Tabrizi SJ. Prion degradation pathways: potential for therapeutic intervention. Mol Cell Neurosci. 2015;66:12-20.

23. Halliday M, Mallucci GR. Review: modulating the unfolded protein response to prevent neurodegeneration and enhance memory. Neuropathol Appl Neurobiol. 2015;41:414-27.

24. Heiseke A, Aguib Y, Riemer C, Baier M, Schatzl HM. Lithium induces clearance of protease resistant prion protein in prioninfected cells by induction of autophagy. J Neurochem. 2009;109:25-34.

25. Krammer C, Vorberg I, Schatzl HM, Gilch S. Therapy in prion diseases: from molecular and cellular biology to therapeutic targets. Infect Disord Drug Targets. 2009;9:3-14.

26. Boellaard JW, Kao M, Schlote W, Diringer H. Neuronal autophagy in experimental scrapie. Acta Neuropathol. 1991;82:225-8.

27. Boellaard JW, Schlote W, Tateishi J. Neuronal autophagy in experimental Creutzfeldt-Jakob's disease. Acta Neuropathol. 1989;78:410-8.

28. Liberski PP, Sikorska B, Bratosiewicz-Wasik J, Gajdusek DC, Brown P. Neuronal cell death in transmissible spongiform encephalopathies (prion diseases) revisited: from apoptosis to autophagy. Int J Biochem Cell Biol. 2004;36:2473-90.

29. Sikorska B, Liberski PP, Giraud P, Kopp N, Brown P. Autophagy is a part of ultrastructural synaptic pathology in Creutzfeldt-Jakob disease: a brain biopsy study. Int $\mathrm{J}$ Biochem Cell Biol. 2004;36:2563-73.

30. Schatzl HM, Laszlo L, Holtzman DM, Tatzelt J, DeArmond SJ, Weiner RI, et al. A hypothalamic neuronal cell line persistently infected with scrapie prions exhibits apoptosis. J Virol. 1997;71:8821-31.

31. Lopez-Perez O, Otero A, Filali H, Sanz-Rubio D, Toivonen JM, Zaragoza P, et al. Dysregulation of autophagy in the central nervous system of sheep naturally infected with classical scrapie. Sci Rep. 2019;9:1911.

32. Lopez-Perez O, Bolea R, Marin B, Badiola JJ, Martin-Burriel I. Autophagy impairment in highly prion-affected brain areas of sheep experimentally infected with atypical scrapie. Vet Microbiol. 2019;233:78-84.

33. Laude H, Vilette D, Le Dur A, Archer F, Soulier S, Besnard N, et al. New in vivo and ex vivo models for the experimental study of sheep scrapie: development and perspectives. C R Biol. 2002;325:49-57.

34. Schulz-Schaeffer WJ, Tschoke S, Kranefuss N, Drose W, HauseReitner D, Giese A, et al. The paraffin-embedded tissue blot detects $\operatorname{PrP}(\mathrm{Sc})$ early in the incubation time in prion diseases. Am J Pathol. 2000;156:51-56.

35. Fraser H, Dickinson AG. The sequential development of the brain lesion of scrapie in three strains of mice. J Comp Pathol. 1968;78:301-11.

36. Vidal E, Acin C, Foradada L, Monzon M, Marquez M, Monleon $\mathrm{E}$, et al. Immunohistochemical characterisation of classical scrapie neuropathology in sheep. J Comp Pathol. 2009;141:135-46.
37. Westaway D, DeArmond SJ, Cayetano-Canlas J, Groth D, Foster D, Yang SL, et al. Degeneration of skeletal muscle, peripheral nerves, and the central nervous system in transgenic mice overexpressing wild-type prion proteins. Cell. 1994;76: 117-29.

38. Niklaus M, Adams O, Berezowska S, Zlobec I, Graber F, SlottaHuspenina J, et al. Expression analysis of LC3B and p62 indicates intact activated autophagy is associated with an unfavorable prognosis in colon cancer. Oncotarget. 2017;8:54604-15.

39. Jeong JK, Park SY. Neuroprotective effect of cellular prion protein (PrPC) is related with activation of alpha7 nicotinic acetylcholine receptor (alpha7nAchR)-mediated autophagy flux. Oncotarget. 2015;6:24660-74.

40. Pircs K, Nagy P, Varga A, Venkei Z, Erdi B, Hegedus K, et al. Advantages and limitations of different p62-based assays for estimating autophagic activity in Drosophila. PLoS ONE. 2012;7: e44214.

41. Kuusisto E, Salminen A, Alafuzoff I. Ubiquitin-binding protein p62 is present in neuronal and glial inclusions in human tauopathies and synucleinopathies. Neuroreport. 2001;12:2085-90.

42. Kuusisto E, Kauppinen T, Alafuzoff I. Use of p62/SQSTM1 antibodies for neuropathological diagnosis. Neuropathol Appl Neurobiol. 2008;34:169-80.

43. Zatloukal K, Stumptner C, Fuchsbichler A, Heid H, Schnoelzer $\mathrm{M}$, Kenner L, et al. p62 Is a common component of cytoplasmic inclusions in protein aggregation diseases. Am $\mathrm{J}$ Pathol. 2002;160:255-63.

44. Nagaoka U, Kim K, Jana NR, Doi H, Maruyama M, Mitsui K, et al. Increased expression of p62 in expanded polyglutamineexpressing cells and its association with polyglutamine inclusions. J Neurochem. 2004;91:57-68.

45. Homma T, Ishibashi D, Nakagaki T, Satoh K, Sano K, Atarashi R, et al. Increased expression of p62/SQSTM1 in prion diseases and its association with pathogenic prion protein. Sci Rep. 2014;4:4504.

46. Mizushima N, Yoshimori T, Levine B. Methods in mammalian autophagy research. Cell. 2010;140:313-26.

47. Thellung S, Scoti B, Corsaro A, Villa V, Nizzari M, Gagliani MC, et al. Pharmacological activation of autophagy favors the clearing of intracellular aggregates of misfolded prion protein peptide to prevent neuronal death. Cell Death Dis. 2018;9:166.

48. Xu Y, Tian C, Wang SB, Xie WL, Guo Y, Zhang J, et al. Activation of the macroautophagic system in scrapie-infected experimental animals and human genetic prion diseases. Autophagy. 2012;8:1604-20.

49. Tanida I. Autophagosome formation and molecular mechanism of autophagy. Antioxid Redox Signal. 2011;14:2201-14.

50. Romanov J, Walczak M, Ibiricu I, Schuchner S, Ogris E, Kraft C, et al. Mechanism and functions of membrane binding by the Atg5Atg12/Atg16 complex during autophagosome formation. EMBO J. 2012;31:4304-17.

51. Gu J, Wang Y, Wang X, Zhou D, Wang X, Zhou M, et al. Effect of the LncRNA GAS5-MiR-23a-ATG3 axis in regulating autophagy in patients with breast cancer. Cell Physiol Biochem. 2018;48:194-207.

52. Xu Y, Tian C, Sun J, Zhang J, Ren K, Fan XY, et al. FBXW7induced mTOR degradation forces autophagy to counteract persistent prion infection. Mol Neurobiol. 2016;53:706-19.

53. Kuma A, Komatsu M, Mizushima N. Autophagy-monitoring and autophagy-deficient mice. Autophagy. 2017;13:1619-28.

54. Pickard MR, Williams GT. Molecular and cellular mechanisms of action of tumour suppressor GAS5 LncRNA. Genes (Basel). 2015;6:484-99.

55. Zhang N, Yang GQ, Shao XM, Wei L. GAS5 modulated autophagy is a mechanism modulating cisplatin sensitivity in NSCLC cells. Eur Rev Med Pharmacol Sci. 2016;20:2271-7. 
56. Li L, Huang C, He Y, Sang Z, Liu G, Dai H. Knockdown of long non-coding RNA GAS5 increases miR-23a by targeting ATG3 involved in autophagy and cell viability. Cell Physiol Biochem. 2018;48:1723-34.

57. Pickford F, Masliah E, Britschgi M, Lucin K, Narasimhan R, Jaeger $\mathrm{PA}$, et al. The autophagy-related protein beclin 1 shows reduced expression in early Alzheimer disease and regulates amyloid beta accumulation in mice. J Clin Invest. 2008;118:2190-9.

58. Martinez-Vicente M, Talloczy Z, Wong E, Tang G, Koga H, Kaushik $S$, et al. Cargo recognition failure is responsible for inefficient autophagy in Huntington's disease. Nat Neurosci. 2010;13:567-76. 